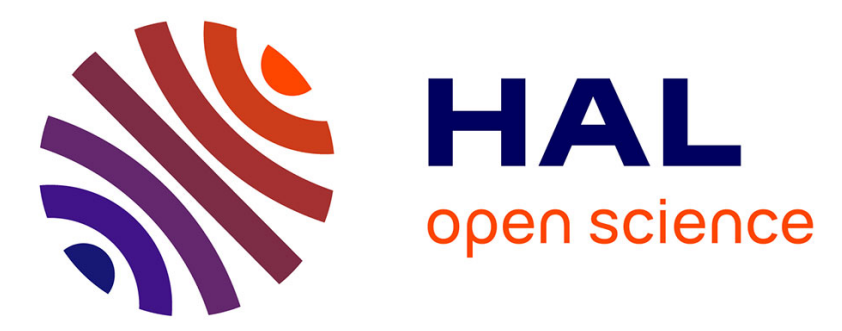

\title{
Conclusion: Evolution pédo-géochimique et interprétation paléobioclimatique de piémont quaternaire garonnais
}

Jacques Hubschman

\section{- To cite this version:}

Jacques Hubschman. Conclusion: Evolution pédo-géochimique et interprétation paléobioclimatique de piémont quaternaire garonnais. Bulletin de l'Association française pour l'étude du quaternaire, 1975, 12 (3-4), pp.211-216. 10.3406/quate.1975.1271 . hal-02735798

\section{HAL Id: hal-02735798 \\ https://hal-univ-tlse2.archives-ouvertes.fr/hal-02735798}

Submitted on 2 Jun 2020

HAL is a multi-disciplinary open access archive for the deposit and dissemination of scientific research documents, whether they are published or not. The documents may come from teaching and research institutions in France or abroad, or from public or private research centers.
L'archive ouverte pluridisciplinaire HAL, est destinée au dépôt et à la diffusion de documents scientifiques de niveau recherche, publiés ou non, émanant des établissements d'enseignement et de recherche français ou étrangers, des laboratoires publics ou privés. 


\section{Conclusion : Evolution pédo-géochimique et interprétation paléobioclimatique de piémont quaternaire garonnais}

\section{Jacques Hubschman}

\section{Abstract}

Conclusions concerning chiefly the soils and geochemical weathering studies in the Garonne Pyrenean piemont. Very developed soils and strong weathered - even with neoformation of kaolinits and gibbsite - pebble alluvions, do not mean the reality of hot ancient - especially pre-Riss - paleoclimatic periods. Soils and weathered formations mean at first their "selfdevelopment" during very long interphases, between relatively short alluvial periods, interphases probably characterized by wet moderate climates.

\section{Citer ce document / Cite this document :}

Hubschman Jacques. Conclusion : Evolution pédo-géochimique et interprétation paléobioclimatique de piémont quaternaire garonnais. In: Bulletin de l'Association française pour l'étude du quaternaire, vol. 12, n³-4, 1975. pp. 211-216;

doi : https://doi.org/10.3406/quate.1975.1271

https://www.persee.fr/doc/quate_0004-5500_1975_num_12_3_1271

Fichier pdf généré le 19/04/2018 


\title{
CONCLUSION
}

ÉVOLUTION PÉDO-GÉOCHIMIQUE ET INTERPRÉTATION

\section{par Jacques HUBSCHMAN}

\begin{abstract}
SUMMARY
Conclusions concerning chiefly the soils and geochemical weathering studies in the Garonne Pyrenean piemont. Very developed soils and strong weathered - even with neoformation of kaolinits and gibbsite - pebble alluvions, do not mean the reality of hot ancient - especially pre-Riss - paleoclimatic periods. Soils and weathered formations mean at first their "self-development" during very long interphases, between relatively short alluvial periods, interphases probably characterized by wet moderate climates.
\end{abstract}

I - Sur le plan stratigraphique général, l'étude pédologique et géochimique, combinée aux données sédimentologiques, géomorphologiques et paléontologiques, permet :

- de préciser l'inventaire purement géomorphologique en définissant 5 grands étages de remblaiements alluviaux quaternaires, chacun d'entre eux se signalant par un degré d'évolution pédo-géochimique particulier ;

- d'assurer de manière satisfaisante la corrélation avec la stratigraphie alpine : ainsi, le Mindel se définit-il comme le plus jeune niveau franchement altéré, argilisé et colmaté, à tendance rubéfiée, homologue du "ferretto" péri-alpin. De même, l'attribution au Donau de la nappe culminante garonnaise s'appuie sur les données établies par $\mathrm{H}$. Alimen et M. Icole dans les Pyrénées occidentales et le Lannemezan. En revanche, l'âge des formations de piémont de type "Lannemezan inférieur", postérieur à la fin du Miocène et antérieur au Donau, ne peut être précisé et reste encore discuté.
II - Concernant le nombre et l'ancienneté des glaciations dans ce secteur des Pyrénées, un fait semble acquis : les Pyrénées ont été envahies au moins à deux reprises par la glace. Au Riss d'abord (Maximum glaciaire), puis au Würm (phase de disjonction de P. Barrère). Par contre, l'existence de glaciations antérissiennes reste encore discutée, notamment par les quaternaristes "pyrénéens". Pour ces derniers, les grandes phases d'alluvionnement antérissiennes correspondraient plutôt à des épisodes rhexistasiques sans doute froids mais à tendance semi-aride, qu'à de véritables périodes glaciaires à la fois très froides et très humides.

III - Du point de vue de la signification paléobioclimatique des sols et des altérations, les informations foumies par les profils invitent plutôt à envisager, depuis le début du Quaternaire, une certaine stabilité des conditions bioclimatiques qui prévalaient durant les "interphases" de pédogénèse ou les interglaciaires (Donau-Günz, Günz-Mindel, Mindel-Riss, Riss-Würm). 
Ces conditions étaient sans doute relativement voisines de celles, tempérées douces et assez humides, qui règnent aujourd'hui dans la région.

Cette façon de voir, dont la portée pourrait s'étendre à des domaines extra-pyrénéens, contredit formellement la thèse plus traditionnelle selon laquelle l'intensité et le mode d'altération des formations prérissiennes traduiraient à coup sûr l'action de bioclimats anciens nettement plus agressifs que ceux d'aujourd'hui. L'interprétation soutenue ici, en revanche, s'inscrit dans la perspective du récent et important courant scientifique qui, depuis quelques années, traverse la Science du sol et dont les travaux de G. Bocquier (1973) et de A. Ruellan $(1971,1973)$, en zone inter- ou subtropicale, peuvent donner un aperçu.

Si l'on revient au piémont garonnais, les principaux arguments qui fondent cette interprétation peuvent être résumés de la façon suivante (J.Hubschman, 1975 a).

1 - Le moteur de l'évolution et de l'altération des remblaiements quaternaires réside essentiellement dans l'épuisement géochimique progressif des alluvions caillouteuses (les limons ne comportant que fort peu de minéraux facilement altérables). Il s'agit d'une sorte de soustraction géochimique (M. Icole, 1973) portant surtout sur les bases et la silice, facilitée par la disposition quasi-horizontale des dépôts et la vidange latérale des solutions percolantes dans les axes de drainage. Chaque étage alluvial évolue ainsi indépendamment des autres et cumule la totalité des actions bioclimatiques (donc, des épuisements géochimiques) postérieures à sa mise en place.

2 - La néogénèse, dès le Mindel, de minéraux kaoliniques et gibbsitique, ne revêt pas en elle-même de signification paléoclimatique précise. Elle procède directement de l'épuisement géochimique progressif évoqué ci-dessus et selon les modalités suivantes :

- D'une part, sitôt atteint un certain degré d'altération, d'argilisation et de colmatage des cailloutis, les alluvions graveleuses s'apparentent à des milieux géochimiques bien lessivés mais à drainage ménagé (G. Millot, 1964). Ainsi, alors que le Riss et surtout le Würm, faiblement altérés et très percolants, ressortissent encore à des milieux géochimiquement "ouverts" et à drainage énergique, les alluvions prérissiennes, fortement argilisées et colmatées, fonctionnent comme des structures déjà moins percolantes, à caractère hydromorphe et riches en nappes d'eau perchées.

. D'autre part, on constate une désaturation en bases des milieux géochimiques de plus en plus poussée du Würm au Donau. Dans le Riss et le Würm, le pH oscille généralement autour de 6. Dans les alluvions du Mindel et du Günz, notamment là où, au sommet des cailloutis, l'altération est maximale, on a vu que les $\mathrm{pH}$ tendent à se rapprocher de 5 . Dans le Donau garonnais et les plateaux de piémont, ils sont presque toujours égaux ou inférieurs à 5 .
Abondance de minéraux aluminosilicatés primaires totalement altérés ou en voie d'altération et libérant des ions $\mathrm{Si}$ et $\mathrm{Al}$; milieu géochimique lessivant mais à drainage ménagé et à hydromorphie générale; $\mathrm{pH}$ acides de l'ordre de 5 dans une part au moins des matrices du Mindel et du Günz et dans l'ensemble des matrices du Donau ... Ce sont là les conditions requises pour la néoformation des kaolinites (G. Millot, 1964), dès le stade d'altération atteint par le Mindel et, a fortiori, par les dépôts antérieurs plus évolués.

La néoformation de gibbsite, moins importante et surtout moins régulière que celle des kaolinites, n'a pas d'autre origine. Mais la genèse et la conservation de la gibbsite requièrent un drainage plus vif et un $\mathrm{pH}$ un peu moins acide que pour les kaolinites. Cet hydroxyde ne peut donc s'élaborer que ponctuellement, au sein de galets altérés plus perméables et moins désaturés que la matrice environnante (M. Icole, 1973). La gibbsite disparaît d'ailleurs pratiquement dans le Donau, plus acide et dont les galets gneisso-granitiques ou ophitiques sont eux-mêmes totalement argilisés et intégrés à la matrice.

Ajoutons enfin que l'abondance et surtout le degré de cristallinité des minéraux kaoliniques néoformés sont évidemment en relation directe avec le $\mathrm{pH}$ et le degré de désaturation en bases, donc avec l'intensité de l'altération globale. On s'explique ainsi qu'abondance et cristallinité de ces minéraux, d'une part, se renforcent du Mindel au Donau, d'autre part, diminuent avec la profondeur dans les cailloutis du Mindel et du Günz (dont les horizons profonds sont de moins en moins altérés et désaturés en bases).

3.-La dynamique du fer traduit avant tout un ensemble de processus secondaires à l'altération globale (qui libère le fer) et à la position dans les toposéquences et au sein des profils (position qui commande la redistribution $d u$ fer).

- La rubéfaction, au demeurant souvent mal exprimée et brouillée par l'hydromorphie, est déjà en germe dans nombre de faciès rissiens, voire würmiens. Dans les alluvions plus anciennes, elle se développe en relation avec les quantités importantes de fer libérées par l'altération des ferromagnésiens et avec le site local ou ponctuel (au sein des profils) de drainage et de redistribution du fer. Ses implications paléoclimatiques sont médiocres, sinon négligeables dans la région considérée.

- Les cuirassements ferrugineux, on l'a vu, sont nettement favorisés par le colmatage poussé du sommet des alluvions caillouteuses prérissiennes, par ailleurs fortement altérées et riches en fer libéré. Ces formations s'apparentent plus ou moins à des "cuirasses de nappe". Elles reflètent surtout le régime hydrique, ancien et actuel, des profils, leur structure sédimentologique et leur position dans les toposéquences.

4 - Les profils associés aux 5 grands étages quaternaires d'alluvions garonnaises matérialisent aujourd'hui 
dans l'espace les différentes étapes chronologiques d'une même séquence évolutive. Cette séquence évo. lutive traduit en quelque sorte le développement "spontané" et auto-entretenu des profils au cours du Quaternaire, dès lors que les remblaiements étaient soumis, pendant de longues durées, à des conditions bioclimatiques modérées mais suffisantes pour perpétuer et intensifier l'évolution (température, humidité, foumiture d'acides humiques....).

Certes, il est clair que le rythme de cet "autodéveloppement" (G. Bocquier 1973) a dû connaître, depuis l'aube du Quaternaire, un certain nombre de variations "positives" ou "négatives". Ainsi, durant les phases plus froides et/ou plus sèches au cours desquelles se déposait une nouvelle génération d'alluvions, les remblaiements antérieurement mis en place évoluaient sans doute moins rapidement que pendant les interphases (ou les interglaciaires). Mais ces variations vers le froid ou le doux, l'humide ou le sec, s'intègrent parfaitement au modèle présenté ici.

L'une des conséquences importantes de ce modèle, on l'a vu, est d'ordre paléoclimatique : les profondes évolutions qui marquent les alluvions quaternaires prérissiennes n'impliquent plus la traditionnelle invocation à des paléoclimats anciens nettement plus agressifs que ceux d'aujourd'hui. Un certain nombre d'indices tendent d'ailleurs à suggérer plutôt le contraire : part importante des structures à 3 couches dans les cortèges de minéraux argileux; reliquats de plagioclases dans les cailloutis altérés du Mindel et du Günz ; sans parler des limons prérissiens dont l'évolution évoque tout à fait une pédogénèse sous bioclimats tempérés humides. Enfin, il n'existe aucun indice paléontologique véritablement probant qui plaide en faveur de climats interglaciaires ou d'interphases, sensiblement plus agressifs - nettement plus chauds, par exemple - que ceux d'aujourd'hui. Faunes et flores "chaudes" nordpyrénéennes ne constituent apparemment qu'un héritage du Tertiaire, qui perdure quelque peu au Quaternaire et s'éteint peu à peu avec le temps. A cet égard, la flore du Mindel-Riss de Montmaurin (M. Girard, 1973) traduit un environnement bioclimatique tout à fait comparable à celui d'aujourd'hui. S'il est hasardeux, faute de données chronologiques précises, d'interpréter la signification paléoclimatique du complexe faunistique étudié par A. Clot à Montoussé (cf. p. 205), il reste que les indications foumies par le gisement de Mauran, vraisemblablement Riss-Würm, doivent être examinées avec une certaine prudence (cf. p. 171). En effet, la présence possible de quelques pollens d'espèces thermophiles comme l'Olivier ou un Pistachier, doit être replacée dans le contexte des Petites Pyrénées, calcaires et compartimentées en multiples facettes écologiques extrêmement contrastées : aujourd'hui encore, le Chêne vert avec son cortège floristique latéméditerranéen, en soulane ou en secteur protégé, pousse à proximité immédiate du Hêtre...
5-Sols et profils d'altération quaternaires sont acutellement bien "vivants". Leur évolution se poursuit encore parfaitement de nos jours. Altération, soustractions géochimiques, transformations et néoformations argileuses, dégradation hydromorphe et redistribution du fer ... Tous ces processus continuent d'évidence à fonctionner aujourd'hui.

C'est pourquoi, contrairement à l'usage, les profils pédologiques développés dans les nappes quaternaires garonnaises ne sont pas assimilés à des paléosols. Cette expression reste en effet très ambigüe et évoque beaucoup trop des formations "fossiles" qui témoigneraient de conditions bioclimatiques révolues et fort différentes de celles d'aujourd'hui, point de vue traditionnel que nous ne partageons absolument pas pour ce qui est du piémont nord-pyrénéen. Pour ces vieux sols, en revanche, - et par opposition aux paléosols significatifs d'un environnement passé spécifique -, nous avons suggéré l'emploi d'un néologisme : les "archéosols" désigneraient ainsi ces profils dont la pédogénèse et l'altération, toujours fonctionnelles de nos jours, ont commencé à une époque très reculée, déterminant éventuellement l'apparition de caractères très différents - souvent d'allure plus ou moins "tropicale" - de ceux qui marquent les profils de génèse récente ou actuelle.

\section{IV - Enfin, les interprétations précédentes débouchent naturellement sur un modèle chronologique et bioclimatique de l'évolution quaternaire dans le piémont nord-pyrénéen.}

Si l'on considère en effet que l'altération des nappes alluviales prérissiennes résulte d'abord de très longues évolutions sous bioclimats modérés, le Quaternaire doit alors être envisagé, depuis le Donau, comme une période marquée essentiellement par la stabilité des paysages (biostasie de H. Erhart, 1967), avec son corrélat majeur: le développement de pédogénèses et d'altérations relativement actives sous climats suffisamment humides et doux. Compte tenu des ordres de grandeur chronologiques du Pléistocène (J.Chaline, 1972), ceci implique que les phases rhexistasiques (glaciaires ou non) d'alluvionnement qui, à 4 reprises depuis la fin du Donau, sont venues rompre cette monotone stabilité, ont dû être considérablement plus brèves, à l'échelle du Quaternaire, que les interphases biostasiques. C'est l'espacement dans le temps de ces phases d'alluvionnement qui explique le contraste plus ou moins vif entre l'évolution pédo-géochimique de 2 nappes alluviales successives. Dans le piémont garonnais, ce contraste est maximal entre le Mindel et le Riss d'une part; entre le Donau et le Günz d'autre part. Donau-Günz et Mindel-Riss représentent ainsi les deux plus longues périodes biostasiques tempérées dans le piémont, comme le schématise la figure 22. Dans ce schéma, les épisodes Donau, Günz et Mindel, figurés ici comme semi-arides, pourraient tout aussi bien être représentés, pour les partisans de glaciations préris- 


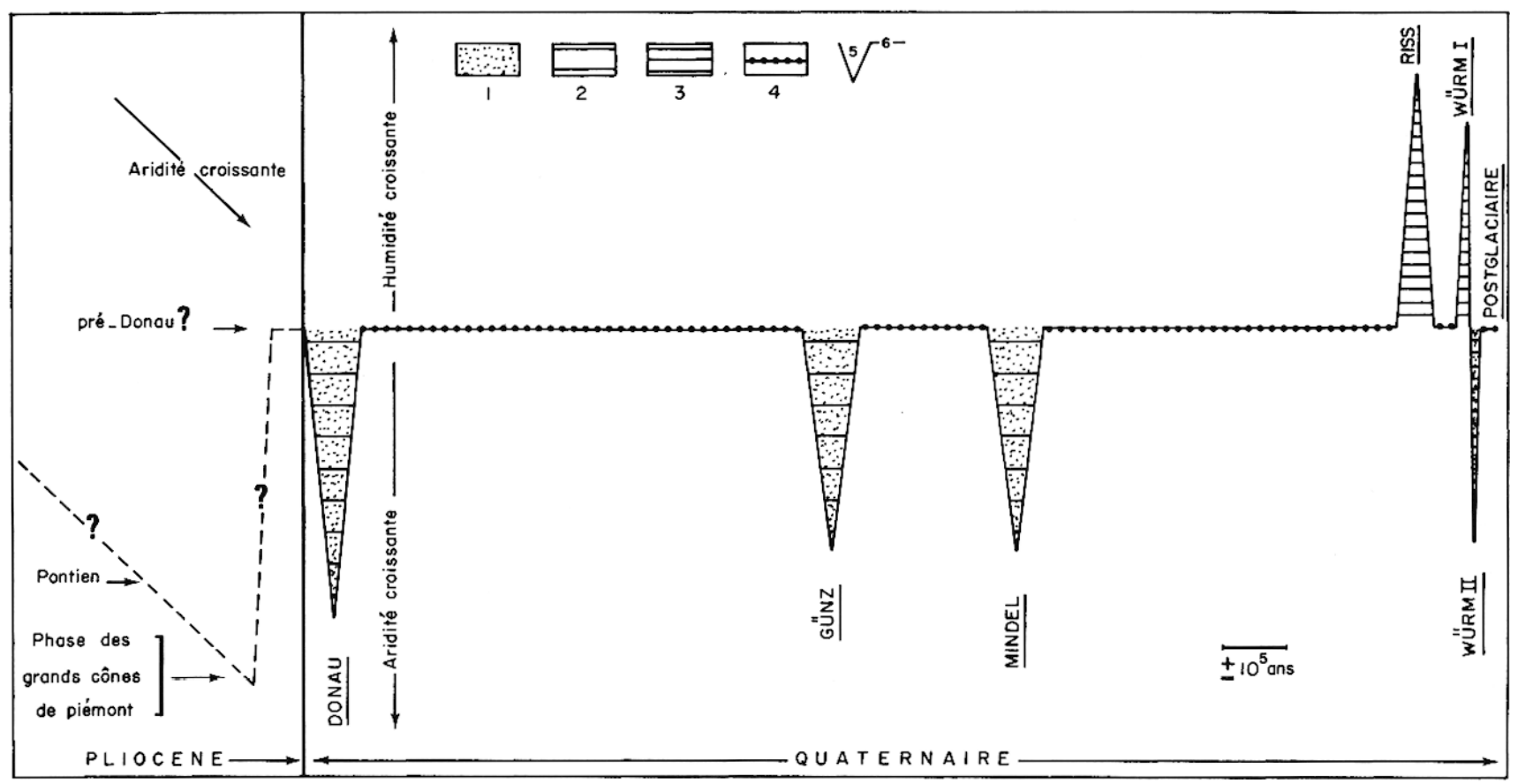

Fig. 22 - Modèle schématique de l'évolution morphoclimatique plio-quaternaire dans le piémont nord-pyrénéen.

1. Episodes semi-arides. 2. Episodes à froid modéré. 3. Episodes à froid vif (glaciations). 4. Episodes tempérées. 5. Phases d'érosion et de remblaiement (rhexistasie). 6. Phases de creusement des cours d'eau et de pédogenèse (biostasie). L'unité de temps $10^{5}$ ans ne concerne que le Quaternaire.

Au cours du Néogène, la tendance aride des climats se renforce et culmine lors de la phase d'épandage des cônes de piémont. Le Donau, peut-être précédé d'un épisode plus humide de creusement (pré-Donau), marque le début du Quaternaire. Celui-ci apparaît globalement comme une longue période biostasique tempérée, entrecoupée de 5 brefs mais efficaces épisodes morphogéniques, dont seuls les deux derniers corresondent à des périodes glaciaires, très rapprochées l'une de l'autre.

siennes, -comme des périodes glaciaires. Dans cette même figure, l'espacement chronologique ne vise à donner que des ordres de grandeur relatifs : il repose sur les estimations actuelles les plus courantes (in J. Chaline 1972) et sur les datations K/A effectuées dans les épandages volcaniques languedociens (J. Barrière, 1969).

\section{BIBLIOGRAPHIE}

Abréviations :

B.A.F.E.Q., Bulletin de l'AFEQ.

B.A.F.E.S., Bulletin de l'Association Française pour l'Etude du Sol.

B.S.C.G.F., Bulletin du Service de la Carte Géologique de France. France.

B.S.G.F., Bulletin de la Société Géologique de

B.S.H.N. Toulouse, Bulletin de la Société d'Histoire Naturelle de Toulouse.

C.R. Ac. Sc., Comptes-rendus de l'Académie des Sciences, Paris.
C.R.S.G.F., Comptes-rendus des séances de la Société Géologique de France.

R.G.P.S.O., Revue Géographique des Pyrénées et du Sud-Ouest, Toulouse.

Alimen H., 1964. - Le Quaternaire des Pyrénées de la Bigorre. Mém. Serv. Carte géol. Fr., Paris, 394 p.

Astre G., 1928. - Quartz à érosion éolienne dans le loess toulousain. B.S.H.N. Toulouse, 57, p. 218-222.

Astre G., 1952. - Faunule malacologique à la base de l'argile grumeleuse de Grépiac. Joumal de Conchyologie (Paris), 92, $\mathrm{n}^{\circ}$ 2, p. 61-64.

Astre G., 1953a. - L'argile grumeleuse de coulière, couverture quaternaire du haut Terrefort toulousain. Bull. Sect. Sciences Com. Trav. Hist. et Scient. (Actes $78^{e}$ Cong. Soc. Sav. Toulouse), p. 65-71.

Astre G., 1953b. - Mastodonte de Bourg-Saint-Bernard et érosions miocènes dans le bassin sous-pyrénéen. B.S.G.F., (6), 3, p. 253-260.

Astre G., 1956. - L'argile grumeleuse sur le loess, aux pentes des coteaux de rive gauche de l'Hers. B.S.G.F., (6), 6, p. 371-380.

Astre G., 1959. - Terrains stampiens du Lauragais et du Tolosan, B.S.H.N. Toulouse, 94, p. 8-168. 
Astre G., 1967. - Elephas trogontherii dans les graviers de Palaminy, B.S.H.N. Toulouse, 103, p. 19-21.

Astre G., 1972. - Le loess de Bellevue et la formation de ses "poupées". B.S.H.N. Toulouse, 108, p. 115-124.

Barrère P., 1953. - Equilibre glaciaire actuel et quaternaire dans l'Ouest des Pyrénées centrales. R.G.P. S.O., XXIV, 2, p. 116-134.

Barrère P., 1963. - La période glaciaire dans l'Ouest des Pyrénées centrales franco-espagnoles. B.S.G.F., (7), 5, p. 516-626.

Barrière J., 1969. - Le volcanisme d'Agde à Valros. Livret-guide excursion C 14, Languedoc, Provence, Côte d'Azur, $8^{\mathrm{e}}$ Cong. INQUA, Paris, p. 14-20.

Bégon J.C., 1972. - Aspects micromorphologiques de la génèse des sols de boulbène. B.A.F.E.S., 1-2, p. 3348 .

Bégon J.C. et Jamagne M., 1972. - Sur la génèse de sols limoneux hydromorphes en France. C.R. Commissions $V$ et VI Ass. Int. Science du Sol, Stuttgart 1971, p. 307-318.

Bocquier G., 1973. - Genèse et évolution de deux toposéquences de sols tropicaux du Tchad. Interprétation biogéodynamique. Mém. O.R.S.T.O.M. n ${ }^{\circ} 62$, $350 \mathrm{p}$.

Boule M., 1895. - Le plateau de Lannemezan et les alluvions anciennes des hautes vallées de la Garonne et de la Neste. B.S.C.G.F., 6, $\mathrm{n}^{\circ} 43$, p. 447-469.

Breuil H., 1937. - Terrasses et quartzites taillés de la haute vallée de la Garonne. Bull. Soc. Préhist. France, 2, p. 1-27.

Carcenac C., Coincon R. et Taillefer F., 1969. - Carte géomorphologique du Mas-d'Azil sud-est (notice). R.G.P.S.O., 40, 4, p. 329-351.

Cavaille A., 1951. - Les sols de boulbène de l'Aquitaine et les climats quaternaires. R.G.P.S.O., XXII, 2-3, p. 199-206.

Cavaille A., 1965. - Les unités morphologiques des basses plaines de la Garonne. R.G.P.S.O., XXXVI, 3, p. 243-278.

Cavaille A., 1969a. - Formations superficielles et sols des coteaux molassiques au sud-est de Toulouse. Livret-guide excursion A 6, Pyrénées orient. et cent., Roussillon, Languedoc occ., $8^{e}$ Cong. INQUA, Paris, p. 16-23.

Cavaille A., 1969b. - Les terrasses de la Garonne à l'ouest de Toulouse. Livret-guide excursion A 6, Pyrénées orient. et cent., Roussillon, Languedoc occ., $8^{\mathrm{e}}$ Cong. INQUA, Paris, p. 10-16.

Chaline J., 1972. - Le Quaternaire. L'histoire humaine dans son environnement. Doin, édit., Paris, 338 p.

Crouzel F., 1957. - Le Miocène continental du Bassin d'Aquitaine. B.S.C.G.F., 54, n 248,264 p.
Crouzel F., Hubschman J. et Revel J.C., 1972. Indices multiples de pédogénèse dans le Miocène continental aquitain. C.R. Ac. Sc., D, 274, p. 2145-2148.

Enjalbert H., 1960. - Les pays aquitains. Le modelé et les sols. Imp. Bière, Bordeaux, 1, 618 p.

Erhart H., 1956. - La génèse des sols en tant que phénomène géologique. Masson et $\mathrm{Cie}$, édit., Paris, 90 p. $2^{e}$ édit., 1967,117 p.

Faucher D., 1931. - Note sur la dissymétrie des vallées de l'Armagnac. B.S.H.N. Toulouse, 56, p. 262-268.

Girard M., 1973. - La brèche à Machairodus de Montmaurin (Pyrénées centrales). B.A.F.E.Q., 3, p. 195-209.

Harle E., 1895. - Observations sur les alluvions de la Garonne dans la région de Toulouse. B.S.G.F., 23, p. 409-503.

Hubschman J., 1972a. - Observations pédologiques sur le Quaternaire du Lauragais toulousain. B.A.F.E.Q., 1, p. 3149 .

Hubschman J., 1972b. - L'âge de la basse plaine garonnaise, des Pyrénées au confluent du Tarn. C.R. Ac. Sc., D, 275, p. 651-653.

Hubschman J., 1973. - Etablissement, par l'étude des faciès d'altération, d'un schéma stratigraphique du Quaternaire garonnais et ariégeois. C.R. Ac. Sc., D, 277 , p. $753-755$.

Hubschman J., 1975a. - Morphogénèse et pédogénèse quaternaires dans le piémont des Pyrénées garonnaises et ariégeoises. Thèse Doct. Etat, Toulouse. Edit. Honoré Champion, Paris, 745 p.

Hubschman J., 1975b. - Le lehm de ToulouseRangueil : signification pédologique et implications géochronologiques. Actes $96^{e}$ Cong. Nat. Soc. Savantes, Sect. Géog., p. 51-60.

Hubschman J. et Le Ribault L., 1972. - Détermination, par exoscopie des quartz, de l'origine des limons quaternaires dans le sud-est du Bassin Aquitain. C.R. Ac. Sc., D, 275, p. 1477-1480.

Icole M., 1969a. - Age et nature de la formation dite de Lannemezan. R.G.P.S.O., 40, 2, p. 157-170.

Icole M., 1969b. - Le plateau de Lannemezan. LivretGuide excursion $A 68^{\mathrm{e}}$ Cong. INQUA, Paris, p. 31-37.

Icole M., 1973. - Géochimie des altérations dans les nappes d'alluvions du piémont occidental nordpyrénéen. Essai de paléopédologie quaternaire. Thèse Sciences Paris, multigr., 328 p.

Lagasquie J.J., 1971. - L'évolution quaternaire du Piémont pyrénéen et des vallées de l'Arbas et du bas Salat. B.S.H.N. Toulouse, 107, p. 125-134.

Méroc L., 1953. - La conquête des Pyrénées par l'Homme. Act. $1^{e}$ Cong. int. spéléo., 4, p. 33-51. 
Méroc L., 1969a. - Les alluvions de la basse vallée du Volp, leurs limons et leurs industries préhistoriques. Livret-guide excursion A 6, Pyrénées orient. et cent., Roussillon, Languedoc occ., $8^{e}$ Cong. INQUA, Paris, p. 24-28.

Méroc L. et Paloume J., 1958. - Nouvelles fouilles à l'Infernet, commune de Clermont-le-Fort (HauteGaronne). B.S.H.N. Toulouse, 43, p. 305-328.

Millot G., 1964. - Géologie des argiles. Masson et Cie, édit., Paris, 499 p.

Mouline M., Birot P. et Paquereau M. 1969. - Le rebord nord-est de la Montagne Noire dans la région de Revel. Livret-guide excursion $A 6$, Pyrénées orient. et cent., Roussillon, Languedoc occ., $8^{e}$ Cong. INQUA, Paris, p. 106-109.

Patin J., 1967. - L'évolution morphologique du plateau de Lannemezan. R.G.P.S.O., 38, 4, p. 325-337.

Richard M., 1946. - Contribution à l'étude du Bassin d'Aquitaine. Les gisements de Mammifères tertiaires. Mém. Soc. Géol. Fr., 24, n 52, 380 p.

Rieucau L., 1957. - Morphologie des terrasses supérieures et moyennes de la Garonne entre les Petites Pyrénées et le Touch. Rev. Géomorpho. Dynam., 5-6, p. 87-92.

Rieucau L., 1958. - Les hauts niveaux des terrasses de la Garonne, entre les Petites Pyrénées et l'Auroue. R.G.P.S.O., XXIX, 4, p. 343-350.

Rieucau L., 1971. - Les arbres fossiles dans la basse plaine de la moyenne Garonne. C.R.S.G.F., 6, p. 317-318.

Ruellan A., 1971. - The history of soils : some problems of definition and interpretation. Paleopedology - Origin, nature and dating of paleosols. Ed. D.H. Yaalon, Int. Soc. Soil, Sc., Israel Univ. Press, Jerusalem, p. 3-12.

Ruellan A., 1973. - Contribution à la connaissance des sols des régions méditerranéenes. Les sols à profil calcaire différencié des plaines de la Basse Moulouya (Maroc oriental). Thèse Sciences Strasbourg, Mém. ORSTOM, $\mathrm{n}^{\circ}$ 54, $304 \mathrm{p}$.

Taillefer F., 1948. - Sur le rôle morphologique des nappes d'alluvions caillouteuses du Bassin d'Aquitaine. C.R. Ac. Sc., 227, p. 978-980.

Taillefer F., 1951. - Le Piémont des Pyrénées françaises. Contribution à l'étude des reliefs de piémont. Privat, édit., Toulouse, 383 p.

Taillefer F., 1969. - Les glaciations des Pyrénées. B.A.F.E.Q. (supplément $8^{\mathrm{e}}$ Congrès INQUA, Paris), p. 19-32.

Taillefer F., 1973. - Le glaciaire de Foix. Ann. Féd. Pyr. Econ. Montagn. (Toulouse), XXIX, s.p.

Tardy Y., 1969. - Géochimie des altérations. Etude des arènes et des eaux de quelques massifs cristallins d'Europe et d'Afrique. Mém. Serv. Carte Géol. Als. Lorr., n ${ }^{\circ} 31,199$ p.

Tavoso A., 1975. - Les terrasses alluviales du bassin du Tarn: éléments de datation archéologiques. B.A.F.E.Q., 1, p. 25-29.

Viers G., 1963. - Les moraines externes de la Cerdagne et du Capcir (Pyrénées orientales, France) et leurs rapports avec les terrasses alluviales. VIth Int. Cong. Quaternary, Warsaw 1961, Lodz, (III), p. 385-393. 